\title{
Evidence for Fibrinogen Mobility on Hydrophobic Surfaces*
}

\author{
Katherine L. Marchin, ${ }^{\dagger}$ Son Phung, and Cindy L. Berrie ${ }^{\ddagger}$ \\ Department of Chemistry, University of Kansas, Lawrence KS 66045, USA
}

(Received 25 April 2005; Accepted 16 May 2005; Published 27 May 2005)

\begin{abstract}
The adsorption of the plasma protein fibrinogen on model hydrophobic surfaces has been explored using atomic force microscopy. Images of fibrinogen adsorbed on both graphite and methyl-terminated self-assembled monolayers are both observed to be highly non-uniform. On both substrates, aggregation and clustering of the fibrinogen molecules is seen. In the case of the graphite surface, we observe a strong preference for adsorption at atomic level steps in the graphite structure. In addition, a region free of protein adsorption is often observed near the step edges. Based on the size of this protein-depleted region, we estimate that the fibrinogen molecules must have diffusion lengths on the order of $\sim 200 \mathrm{~nm}$ under the conditions of these experiments. These results suggest that in the initial stages of adsorption, fibrinogen is quite mobile on a hydrophobic surface.
\end{abstract}

[DOI: $10.1380 /$ ejssnt.2005.173]

Keywords: Atomic force microscopy; Diffusion and migration; Adhesion; Biological aspects of nanostructures; Nanoscale imaging

\section{INTRODUCTION}

The adsorption of proteins onto solid surfaces is important in a number of areas including biosensor fabrication, biocompatibility of implants, and affinity chromatography [1]. The adsorption of proteins is thought to proceed through several steps, including the adsorption of a weakly bound precursor state followed by possibly diffusion and conformational changes that lead to a more tightly bound configuration $[2,3]$. However, the details of each of these stages of the process have not been investigated thoroughly. The conformation and orientation of proteins at the surface is important in the activity of surface-adsorbed proteins and therefore is a critical topic for investigation. Atomic force microscopy provides an excellent approach for probing protein-surface interactions and the conformation of proteins at the single molecule level $[4,5]$.

The plasma protein fibrinogen is a large $(\sim 340 \mathrm{kD})$ oligomeric protein which is abundant in blood plasma $[6,7]$. It has been studied extensively since it is involved in thrombosis (blood-clotting). The conformation and structure of the adsorbed fibrinogen layer are thought to be important in thrombus formation and platelet adhesion [811]. The structure of fibrinogen is trinodular with a length of approximately $47 \mathrm{~nm}[7,12,13]$. Previous work has shown that fibrinogen adsorbs differently to surfaces with different chemistry [14-16]. On some surfaces, fibrinogen shows a tendency to aggregate while on others it adsorbs primarily as isolated single molecules [17]. The kinetics of fibrinogen adsorption at surfaces have been previously investigated as a function of surface chemistry and have shown significant variation [18-20]. While the kinetics of protein adsorption have been investigated, little detail is known about the distribution of fibrinogen molecules on

*This paper was presented at International Symposium on Molecule-Based Information Transmission and Reception - Application of Membrane Protein Biofuction- (MB-ITR2005), Okazaki, Japan, 3-7 March, 2005.

${ }^{\dagger}$ Present address: Midwest Research Institute, Kansas City, MO, USA

$\ddagger$ Corresponding author: cberrie@ku . edu the surface. Recent measurements suggest that conformational differences in the protein are responsible for the variation with surface chemistry $[17,21]$. These conformational changes may lead to changes in the activity of the surface-adsorbed fibrinogen and therefore a detailed understanding of the conformation and structure of the adsorbed protein film is highly desired.

In this work, we investigate the adsorption of fibrinogen on graphite and methyl-terminated self-assembled monolayer films. These two surfaces are chemically quite similar (both hydrophobic) but the graphite surface possesses well-defined atomic level steps that are absent in the case of the self-assembled monolayer system. Fibrinogen films on each of these two substrates are imaged using atomic force microscopy and the distribution of molecules on the two surfaces are compared. Specifically, we observe preferential adsorption near step edges in the case of graphite surfaces and this allows the estimation of a lower limit to the diffusion length of the molecules on these surfaces.

\section{EXPERIMENTAL}

\section{A. Materials and Supplies}

The HOPG graphite samples were purchased from VEECO (Digital Instruments) and cleaved immediately prior to use, and self-assembled monolayers on silicon were prepared as described below. The Si(111) wafers ( $n$-doped, resistivity of $0.001-0.01 \Omega$-cm) were purchased from Virginia Semiconductor. The bovine fibrinogen was obtained from Sigma and used without further purification. Fibrinogen solutions were prepared with concentrations of $\sim 0.1$ to $10 \mu \mathrm{g} / \mathrm{mL}$ in phosphate buffer. Standard phosphate buffers of $\mathrm{pH} 7.0$ and 8.0 and were obtained from Fisher Scientific and VWR respectively. Milli-Q water (resistivity $>18.2 \mathrm{M} \Omega-\mathrm{cm}$ and $\mathrm{pH}=6.0$ ) was used whenever water was required. Chemicals for the monolayer formation including 1-octadecene, 1-dodecene, ammonium fluoride, chloroform, and ammomium fluoride were obtained from either Fisher Scientific or Aldrich and were used without further purification. 


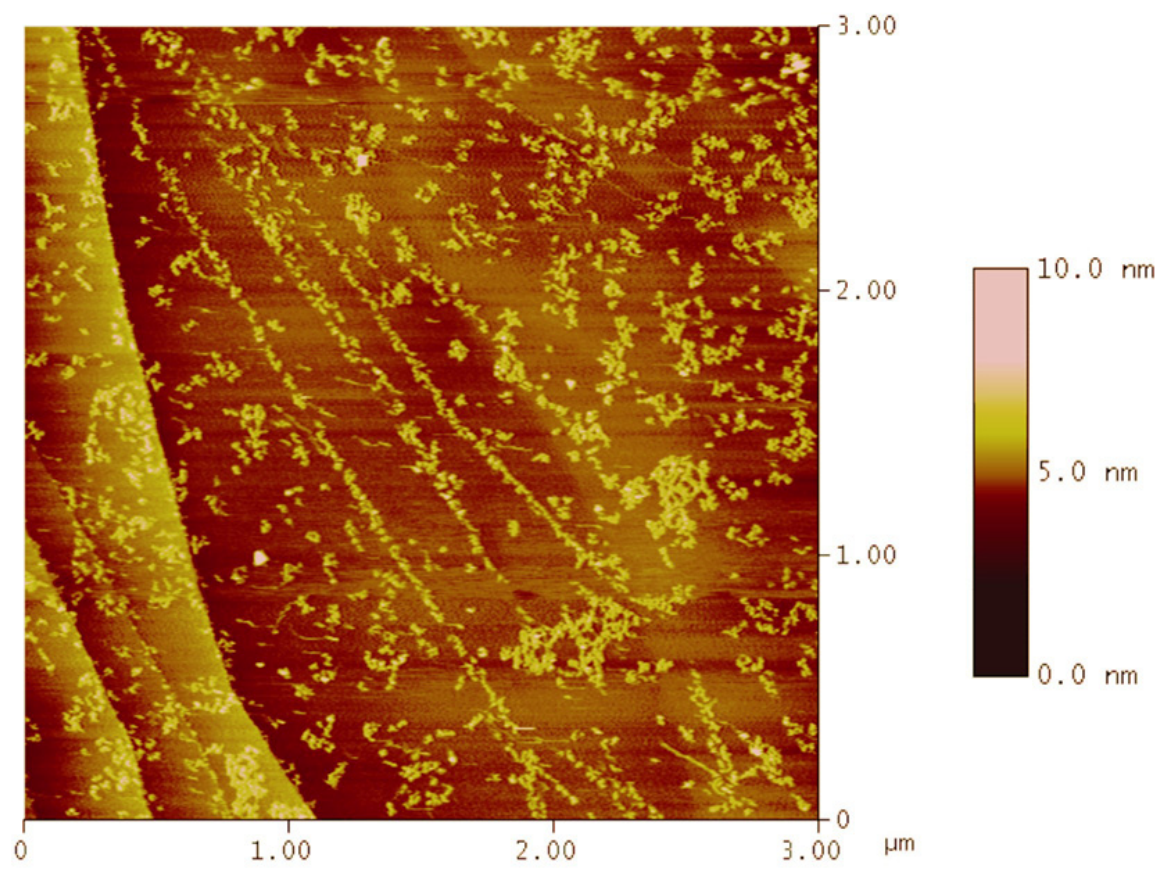

FIG. 1: AFM image of bovine fibrinogen adsorbed from phosphate buffer onto a graphite substrate $(3 \times 3 \mu \mathrm{m})$. The image shows molecules clearly aggregated with large regions on the surface free of adsorbed protein. In addition, protein accumulates preferentially at the step edges.

\section{B. Preparation of self-assembled monolayers}

Self-assembled monolayers were formed following the method of Sung et al. for forming alkyl monolayers on hydrogen-terminated silicon substrates [22]. The dodecene self-assembled monolayer films on $\mathrm{Si}(111)$ were prepared by rinsing the $\mathrm{Si}(111)$ wafers with acetone followed by etching in a saturated solution of ammonium fluoride for $\sim 5 \mathrm{~min}$. The samples were then rinsed briefly in milli-Q water and methanol and allowed to dry in air. The dodecene solution was degassed by bubbling nitrogen through the solution for $\sim 30$ minutes. The solution was then heated to $\sim 170-190^{\circ} \mathrm{C}$. The samples were added to the dodecene solution and held at this temperature for $\sim 1$ hour. The solution was then cooled and the samples removed and rinsed with chloroform. The average $\mathrm{H}_{2} \mathrm{O}$ contact angle for these samples was $95^{\circ}$, consistent with previous measurements [22].

\section{Atomic Force Microscopy}

All of the images presented here were obtained using a VEECO (Digital Instruments) Multimode microscope with a Nanoscope IIIa controller. The images were collected in tapping mode using silicon cantilevers tuned at their resonant frequency. Typical scan rates for data collection were 1-3 Hz. The AFM probe tips were obtained either from VEECO (TESP tapping mode cantilevers) or MikroMasch (NSC12 noncontact cantilevers). Images presented in this paper were collected in air after adsorbing a few drops of protein solution on the substrate for a certain amount of time, rinsing the sample with buffer and/or milli-Q water and dried under a stream of nitrogen gas. The samples were fixed to a magnetic disk for imaging. The images presented here are unfiltered, the only manipulation that has been done is a plane fit correction where needed. We have made no attempt to deconvolute the effect of the probe tip in this data.

\section{Contact Angle}

Static contact angles were measured using a Rame-Hart 100-00 NRL goniometer with the sessile drop method. Contact angles were obtained on $\sim 2 \mu \mathrm{L}$ droplets of milli$\mathrm{Q}$ water on several spots on each sample.

\section{RESULTS AND DISCUSSION}

Figure 1 shows a $3 \times 3 \mu \mathrm{m}$ image of fibrinogen adsorbed on a graphite substrate. The sample was imaged under ambient conditions in tapping mode as described above. There are several features of interest in this image. The molecules appear to be aggregated together on the surface rather than adsorbed as isolated single molecules. This is consistent with our recent work on the conformation of surface-adsorbed fibrinogen [17]. These clusters often appear as extended networks of molecules, some having dendritic-type character. In addition, atomic level steps in the graphite substrate are visible. The molecules show a clear tendency to adsorb near the step edges. This is not surprising since these step edges might act as defect sites on the surface with higher affinity and thereby act as sinks for adsorption. The density of the molecules on the terraces in the vicinity of the step edges is reduced from that farther away from the step edges. In some cases, the 


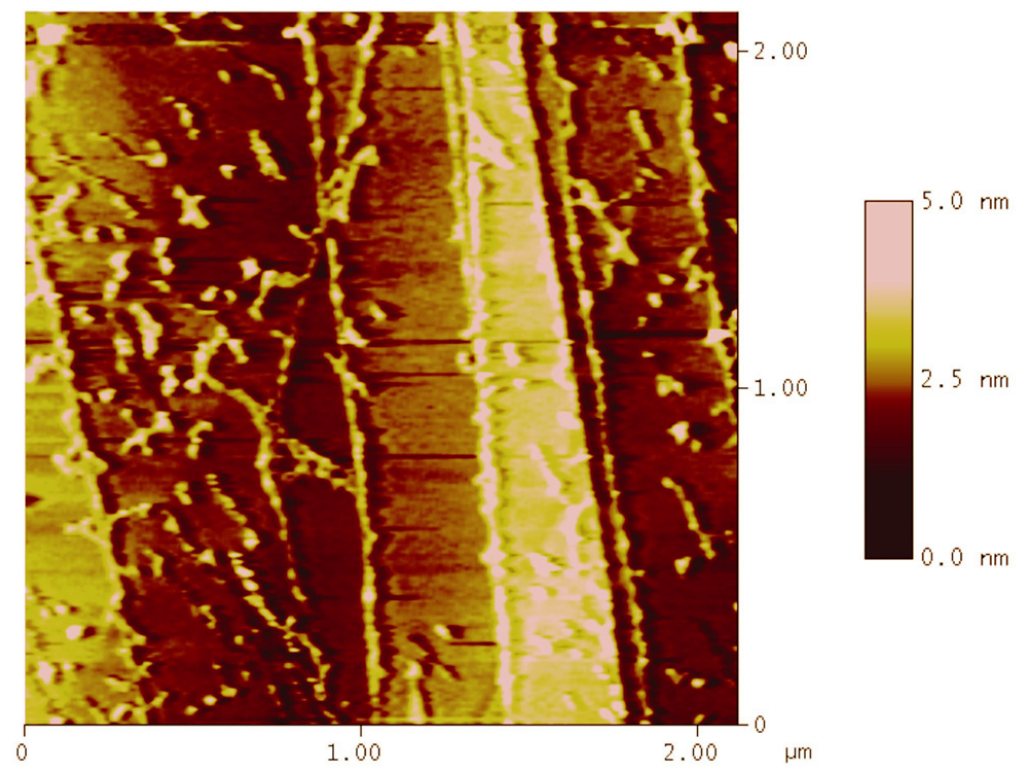

FIG. 2: AFM image of bovine fibrinogen adsorbed on a graphite substrate $(2.1 \times 2.1 \mu \mathrm{m})$. The center terrace in this image is nearly free of protein adsorption, which implies that the protein must be able to diffuse to the step edges of this terrace. In this case, a distance of $\sim 170 \pm 10 \mathrm{~nm}$.

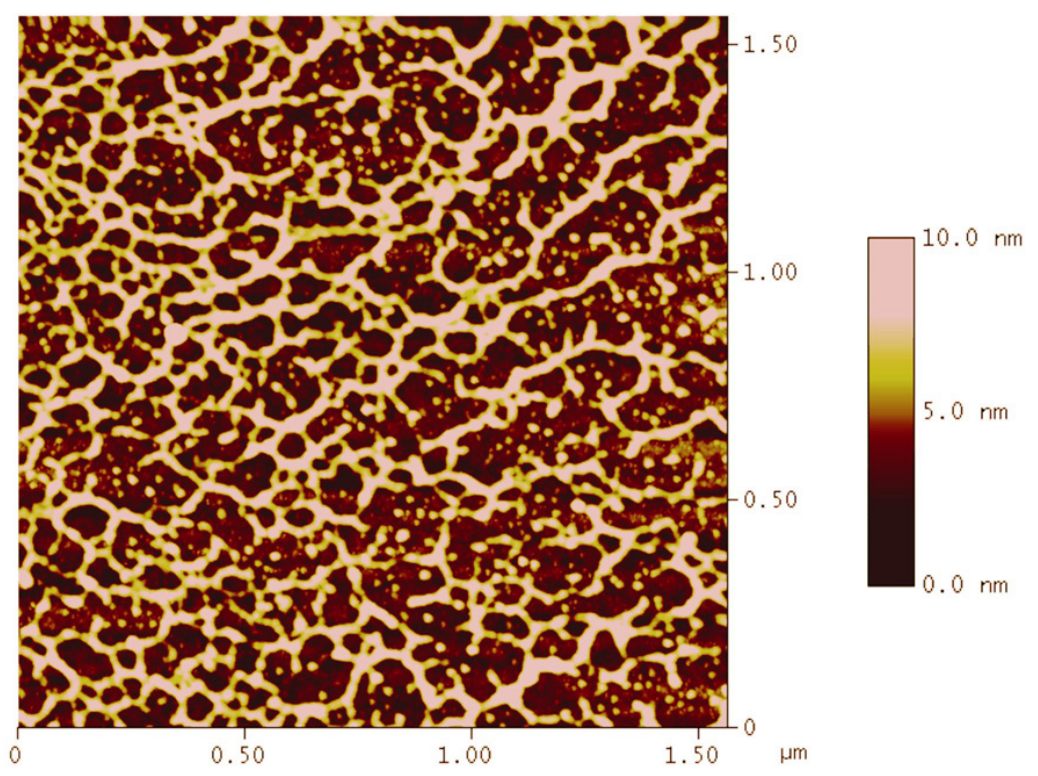

FIG. 3: AFM image of bovine fibrinogen adsorbed on a methyl-terminated, hydrophobic SAM film $(1.6 \times 1.6 \mu \mathrm{m})$. The molecules again appear clustered as in the case of the graphite substrate, but here, there are no sharp step edges and the protein adsorption is more uniform.

terraces are almost completely devoid of adsorbed protein molecules. This is demonstrated by the image shown on Figure 2 where the $\sim 345 \mathrm{~nm}$ wide terrace in the center of the image shows protein adsorbed at the edge, but very little within the terrace region.

The adsorption of fibrinogen on a methyl-terminated alkyl monolayer on $\mathrm{Si}(111)$ is shown in Figure 3. As in the case of the images on the graphite surface presented in Figures 1 and 2, the molecules do appear to be clustered and aggregated on this surface as well. However, since this surface lacks the well-defined atomic level steps that are present on the graphite surface, the adsorption appears to be more uniform over the entire surface. In this case, the coverage of surface-adsorbed fibrinogen is higher than in the case of the graphite images presented above. An image of a similar SAM film with a lower density of fibrinogen adsorbed is shown in Figure 4. From this image, the underlying roughness in the SAM film is visible, but the protein is clearly distinguishable and still displays the clustering mentioned above, even at this lower density. The molecules are able to find one another and form aggregates on the surface even at this fairly low density. 


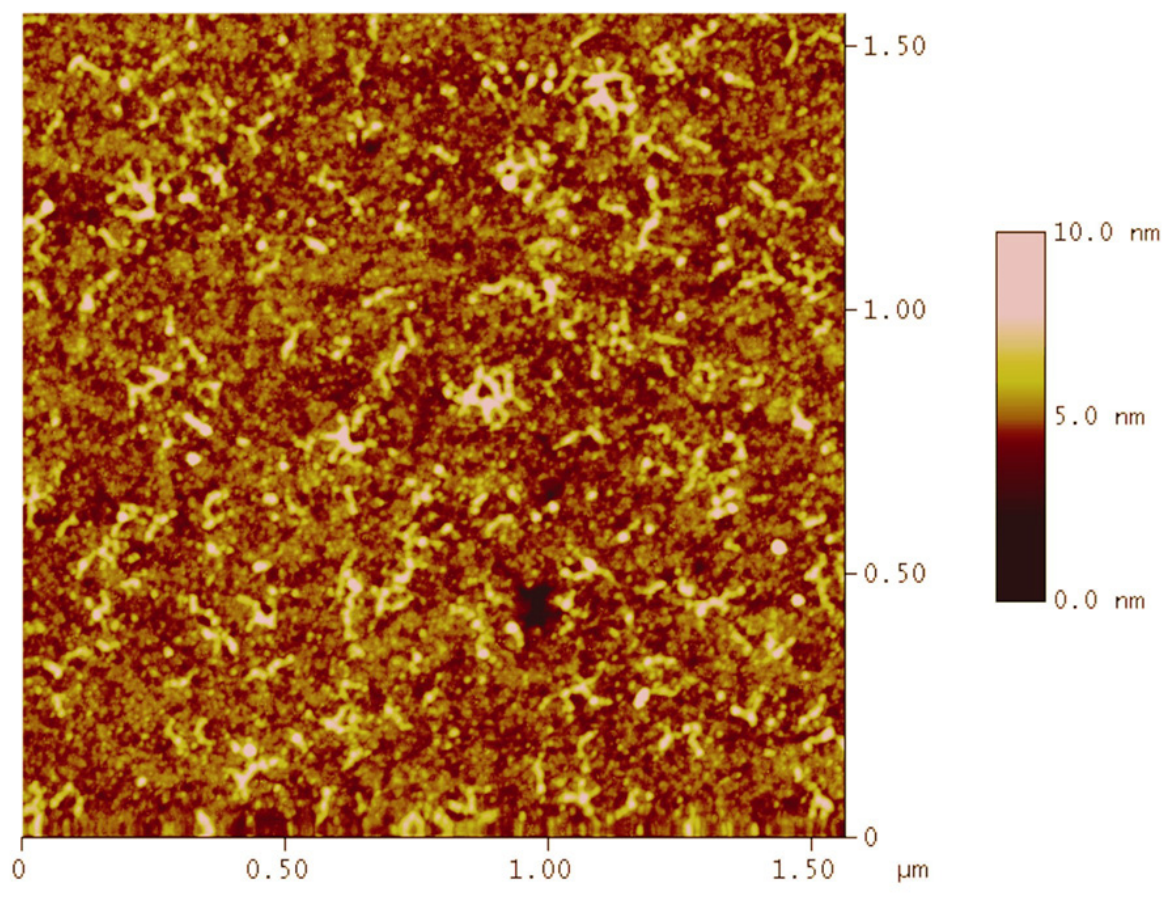

FIG. 4: AFM image of bovine fibrinogen observed on a methyl-terminated SAM surface at a coverage significantly lower than observed in Figure 3. The roughness of the monolayer film is apparent, but there is also still obvious protein adsorption and aggregation.

Assuming that the molecules impinge upon the substrate randomly, there should be no preference for a molecule landing at the step edge on one of these graphite surfaces than on the terraces. Therefore, the fact that we observe preferential adsorption at the step edges with regions of low density nearby indicates that the molecules are able to diffuse on the terraces once they land on the surface in order to get to a step edge. As an example, take the terrace shown in Figure 2. In order for all of the molecules which land on the terrace to make it to the step edge, the molecules which landed in the center of the terrace must diffuse by at least one half the width of the terrace to reach the step edge. In this case, the average terrace width in this image for the clear terrace is $340 \pm 20$ $\mathrm{nm}$. That means that at a minimum the molecules must be able to diffuse $170 \pm 10 \mathrm{~nm}$. This represents a lower limit on the diffusion length because it assumes that the molecules all diffuse with the shortest possible path directly toward the step edge; the average diffusion length may be significantly longer than that predicted from this model. Analysis of this type has been carried out for a number of steps with estimated diffusion lengths ranging from 170 to $670 \mathrm{~nm}$.

Another example of fibrinogen adsorbed on a graphite sample is shown in Figure 5. In this case, there is a large protein depleted region in the center of the image. By measuring the average diameter of this region, we can estimate that for molecules to diffuse out of this region from the center, they would have had to have a diffusion length of one half the diameter, or approximately $280 \pm 50$ $\mathrm{nm}$.

At lower surface coverage, the effects seem even more dramatic. Figure 6 shows an image of a graphite surface in which nearly all of the fibrinogen has accumulated at the step edges. The overall coverage of the fibrinogen as well as the adsorption time seem to be important in determining the overall protein distribution on the surface.

On the SAM films, the molecules appear clustered and aggregated as well, but the surface coverage is more homogeneous and there are not the obvious protein-depleted regions observed in the case of the graphite substrate. This is likely due to the fact that the step edges act as nucleation sites for adsorption and there are not similar preferential adsorption sites available on the SAM films. However, there are well-defined clusters with bare substrate in between them and in order for the molecules to form such clusters, the molecules must initially have some mobility on the surface, just as in the case of the graphite substrate. However, the nucleation of the clusters is more evenly distributed on this surface than on the graphite surface.

Previous investigations of the kinetics of protein adsorption on hydrophobic surfaces have suggested very little diffusion on the surface [20]. However, these experiments were fluorescence recovery after photobleaching experiments that were conducted at some time after the initial adsorption event and do not adequately address the initial mobility of the molecules on the surface. The results obtained here suggest that in the initially adsorbed state, the molecules are quite mobile on the surface and can diffuse a distance on the order of hundreds of nanometers before irreversible adsorption occurs. (Continued imaging of a given region of the surface for several hours shows very little change in protein distribution on the surface.) This initial mobility is not inconsistent with a model of protein adsorption in which the protein initially 


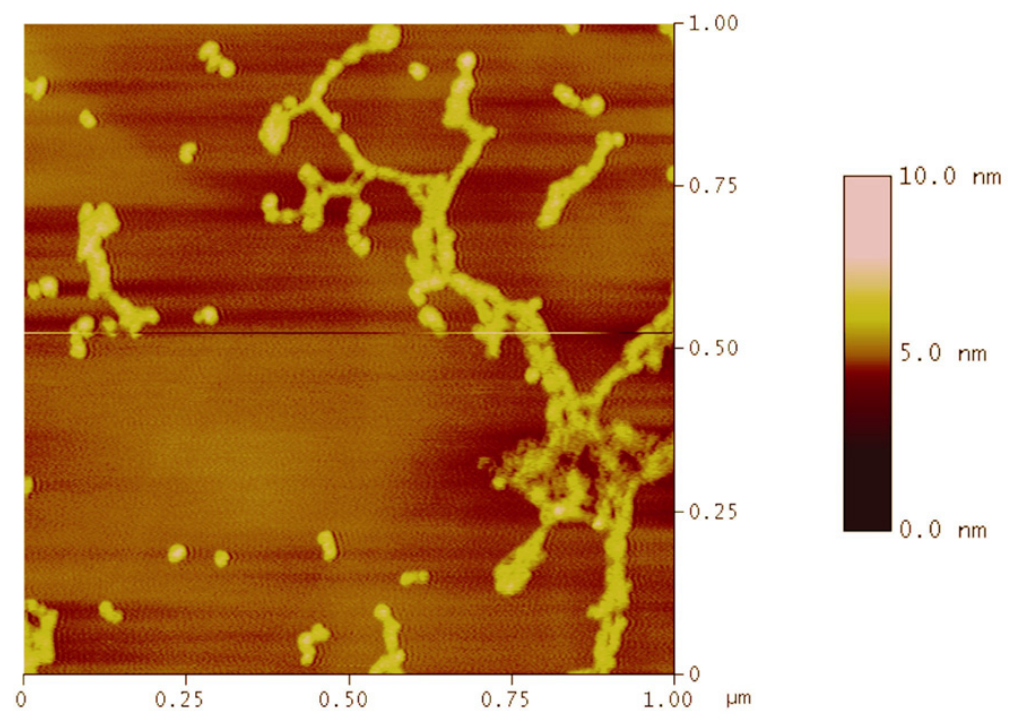

FIG. 5: AFM image of fibrinogen adsorbed on graphite surface $(1 \times 1 \mu \mathrm{m})$. In this case, a clear area is observed in the center of the image which is free of protein adsorption. By measuring the diameter of this area, it is estimated that protein adsorbed on this surface would have had to diffuse $280 \pm 50 \mathrm{~nm}$.

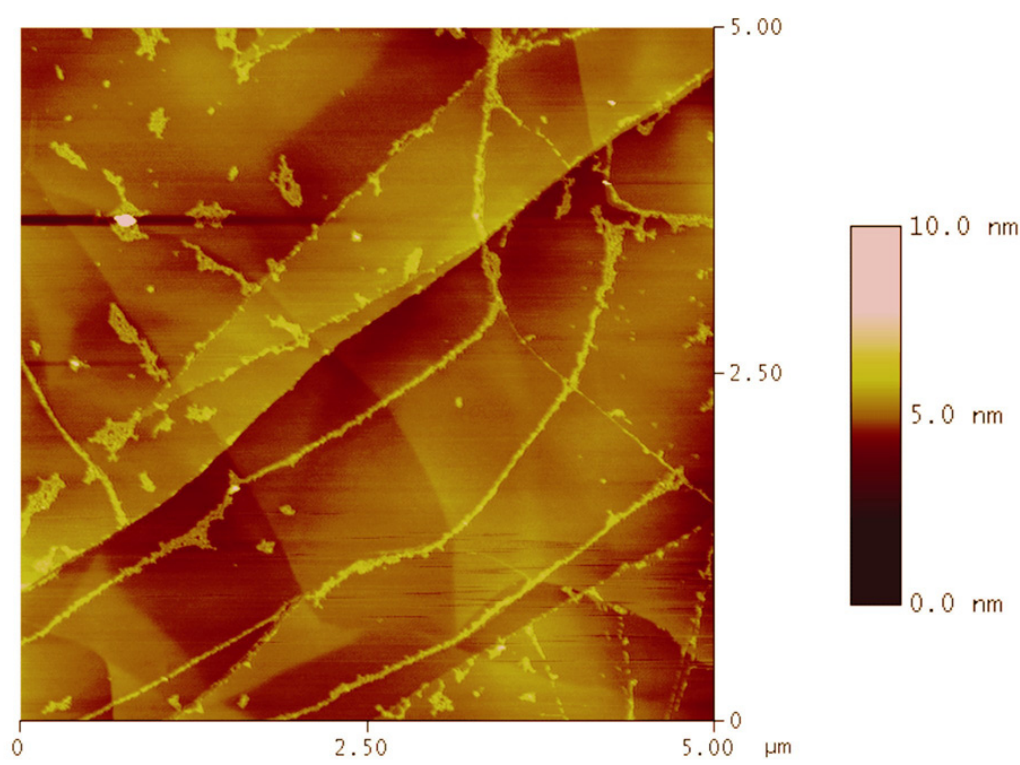

FIG. 6: AFM image of protein adsorbed on graphite surface at low coverage. In this case, the terraces are nearly free of protein adsorption and all of the protein has managed to diffuse to the step edges for adsorption. In this case, the diffusion length of the fibrinogen must be quite large.

adsorbs in a weakly bound state followed at a later time by a transition to a more tightly bound state $[2,3]$. In this case, the weakly bound state must be quite mobile on the surface. Additional evidence for a weakly bound, possibly more mobile state, is provided by experiments that demonstrate the ability of other proteins to displace surface-adsorbed fibrinogen decreases with time as the protein becomes more tightly bound [23]. It is possible that this initial mobility on the surface results from multiple absorption-desorption events at different sites on the surface until the protein is adsorbed into a more tightly bound state and is not the direct result of diffusion on the surface, but in either case the adsorption must occur initially in a relatively weakly bound state.

It is clear from this work that the nanoscale structure of the substrate does play a role in the adsorption of proteins at the surface. In this case, nanoscale steps effectively 'trap' fibrinogen molecules preferentially over sites on the terraces. The mechanism of this is not yet clear. Many nanobiotechnology innovations require the ability to control location and structure of proteins adsorbed at surfaces. These results indicate that some control over protein film structure can be achieved through stepped features on the surface.

This study has only investigated one of the properties that are likely to influence protein film structure at sur- 
faces, namely the influence of atomic level steps. However, there are a number of other factors which almost certainly play a role in how the protein adsorbs and diffuses on the surface including protein concentration, adsorption time, flow conditions, ionic strength, and $\mathrm{pH}$. These factors have not been specifically addressed in this work and are currently being further investigated.

\section{CONCLUSIONS}

We have observed clustering and aggregation of the plasma protein fibrinogen on both graphite and hydrophobic SAM surfaces. The structure of the steps on the graphite substrate seems to dictate protein adsorption, as fibrinogen is observed to preferentially bind at the step edges. Near the steps, large regions with a low density of protein are observed, indicating that the molecules that initially adsorbed on the surface in this region have been able to diffuse away to more favorable binding sites. By measuring the size of these protein-depleted regions, a lower limit of the diffusion length of the fibrinogen of $\sim 200$ $\mathrm{nm}$ is obtained. This suggests a model of fibrinogen adsorption on these surfaces where the initial adsorption of the molecule is into a weakly bound precursor state which can diffuse on the surface before irreversible adsorption (possibly due to a conformational change) occurs, often at an aggregate or step edge.
[1] M. Malmsten, Biopolymers at Interfaces, 2nd Ed. (2003).

[2] T. A. Horbett and J. L. Brash; Proteins at Interfaces I, ACS Symp. Ser. 343, 1 (1987).

[3] T. A. Horbett and J. L. Brash, Proteins at Interfaces II: Fundamentals and Applications, ACS Symp. Ser. 602, 1 (1995).

[4] H. G. Hansma and J. H. Hoh, Annual Review of Biophysics and Biomolecular Structure 23, 115 (1994).

[5] H. G. Hansma and L. Pietrasanta; Current Opinion in Chemical Biology 2, 767 (1998).

[6] G. McManama, J. N. Lindon, M. Kloczewiak, M. A. Smith, J. A. Ware, J. Hawiger, E. W. Merrill and E. W. Salzman; Blood 68, 363 (1986).

[7] L. Feng and J. D. Andrade; ACS Symp. Ser. 602, 66 (1995).

[8] W. G. Pitt, K. Park and S. L. Cooper; Journal of Colloid and Interface Science, 111, 343 (1986).

[9] J. N. Lindon, G. McManama, L. Kushner, E. W. Merrill and E. W. Salzman; Blood 68, 355 (1986).

[10] R. W. Pekala, E. W. Merrill, J. Lindon, L. Kushner and E. W. Salzman; Biomaterials 7, 379 (1986).

[11] V. Balasubramanian, N. K. Grusin, R. W. Bucher, V. T. Turitto and S. M. Slack; J. Biomed. Mater. Res. 44, 253 (1999).

[12] J. W. Weisel, C. V. Stauffacher, E. Bullitt and C. Cohen;
Science 230, 1388 (1985).

[13] R. R. Gorman, G. E. Stoner and A. Catlin; J. Phys. Chem. 75, 2103 (1971).

[14] R. Wigren, H. Elwing, R. Erlandsson, S. Welin and I. Lundstrom; Febs Letters 280, 225 (1991).

[15] P. S. Sit and R. E. Marchant; Thromb. Haemostasis 82, 1053 (1999)

[16] R. E. Marchant, M. D. Barb, J. R. Shainoff, S. J. Eppell, D. L. Wilson and C. A. Siedlecki; Thromb. Haemostasis 77, 1048 (1997).

[17] K. L. Marchin and C. L. Berrie; Langmuir 19, 9883 (2003).

[18] C. F. Wertz and M. M. Santore; Langmuir 17, 3006 (2001).

[19] C. F. Wertz and M. M. Santore; Langmuir 18, 706 (2002).

[20] H. Nygren, S. Alaeddin, I. Lundstroem and K.-E. Magnusson; Biophys. Chem. 49, 263 (1994).

[21] T. C. Ta, M. T. Sykes and M. T. McDermott; Langmuir 14, 2435 (1998).

[22] M. M. Sung, G. J. Kluth, O. W. Yauw and R. Maboudian; Langmuir 13, 6164 (1997).

[23] T. A. Horbett, K. W. Cooper, K. R. Lew and B. D. Ratner; J. Biomater. Sci., Polym. Ed. 9, 1071 (1998). 